\title{
Die Internationalisierung der föderalistischen Bildungspolitik
}

\section{Fritz Osterwalder und Karl Weber}

Es wird danach gefragt, wie sich Akteure, Konstellationen der schweizerischen Bildungspolitik und ihre Programmatik auf die Internationalisierung der Bildungspolitik einstellen. Dabei wird davon ausgegangen, dass sich sowohl zentralisierende wie auch auf Differenz orientierte Akteure der Bildungspolitik an internationalen Projekten beteiligen. Ausgangspunkt der Untersuchung ist dabei die Entwicklung des historischen Gegensatzes zwischen vertikal-koordinierender, stärker vereinheitlichender und horizontal-koordinierender, auf Erhaltung der Differenz ausgerichteter Politik. Während in den meisten politischen Feldern historisch im allgemeinen die zweite Ausrichtung eindeutig dominant war, hat sich in der Bildungspolitik konkurrenziell dazu auch die erste entwickelt. In der Expansionsphase des Bildungssystems in der zweiten Hälfte des 20. Jahrhunderts wird das bildungspolitische Feld zunehmend differenziert. Neue Akteure treten auf und traditionelle Akteure versehen sich mit wissenschaftsorientierten Stäben. Dabei erschöpften sich beide historischen Orientierungen zusehends programmatisch. Das stärkere Auftreten internationaler Akteure, OECD, EU, das Hervortreten internationaler Foren, Konstellationen und Strategien wird sowohl für historisch dezentral, auf Differenz wie auch für historisch auf Vereinheitlichung orientierte Akteure zur gemeinsamen Referenz.

Es wäre übertrieben zu behaupten, die Ergebnisse der PISA-Studie («Programme for International Student Assessment») hätten die schweizerische Bildungspolitik in ihren Grundfesten und ihrem Selbstverständnis verunsichert oder gar erschüttert, obwohl die Resultate dazu durchaus einen Anlass hätten abgeben können. ${ }^{1}$ Überraschend ist eher, mit welcher Unaufgeregtheit in der Schweiz die bildungspolitisch Verantwortlichen wie die kommentierende Öffentlichkeit die eher dürftigen Resultate wahrgenommen und kommentiert haben (Zeitschrift für Bildungswissenschaften, 2003/1, Hagenbüchle, 2002). Überraschend ist die nüchterne Reaktion deswegen, weil in der bildungspolitischen Auseinandersetzung bislang Vielfalt, Dezentralisierung, kantonale Autonomie und Selbstbeurteilung immer, wenn auch mit wechselnden Akzenten, als höchste Priorität be- 
urteilt wurden. Die hohe Akzeptanz des Fremdurteils über das schweizerische Bildungssystems muss noch bedeutender erscheinen, wenn der Anspruch von PISA berücksichtigt wird, einen internationalen Gütemassstab zu bieten, woran fortan die Bildungspolitik auszurichten sei.

Wir fragen nach der Bedeutung dieses Wandels und nach den verschiedenen nationalen und internationalen institutionellen und programmatischen Bedingungen, die ihm zugrunde liegen. ${ }^{2}$ Diese Frage bearbeiten wir in drei Schritten: $\mathrm{Zu}-$ nächst werden die bildungspolitischen Dimensionen expliziert, welche PISA und ähnlichen Studien zu Grunde liegen. Dabei interessieren Fragen der bildungspolitischen Steuerungsabsicht der OECD (Organisation for Economic Cooperation and Development), der Akteurkonstellation, der Vereinheitlichung und der Standardisierung. In einem zweiten Schritt skizzieren wir schematisch das Spannungsfeld des Bildungsföderalismus der Schweiz, der Macht- oder Entscheidungskompetenzverteilung im institutionellen Gefüge und der dabei generierten Programmatik, um auf diesem Hintergrund den Wandel der neuesten Zeit $\mathrm{zu}$ rekonstruieren. Im Vordergrund stehen dabei die unterschiedlichen Akteure und ihre Perspektiven sowie die unterschiedlichen Akteurkonstellationen, die sich auf den verschiedenen Ebenen der bildungspolitischen Entscheide ergeben. Im dritten Schritt werden die Veränderungen der internationalen bildungspolitischen Umwelt ebenso schematisch skizziert. Wir konzentrieren uns dabei auf institutionell sehr unterschiedlich ausgestattete Akteure und Konstellationen, den Europarat, die OECD und die EU. Dabei soll gezeigt werden, welche Akteure mit welcher Programmatik auftreten und welches die Implikationen für die schweizerische Bildungspolitik sind. Schliesslich wird zusammenfassend gefragt, wie diese skizzierten Veränderungen sich aufeinanderbeziehen, bzw. in welchen Formen sich die traditionell föderalistische Bildungspolitik der Schweiz internationalisiert.

Entsprechend der hier entwickelten Fragestellung beziehen wir uns auf eine akteur- und strukturtheoretische Perspektive. Wir gehen davon aus, dass das Handeln der Akteure stets durch ihre Programmatik als auch durch Strukturen bedingt ist, die sowohl Handlungsspielräume eröffnen wie auch solche begrenzen. Diese Strukturen wie auch die Programmatik sind allerdings nicht stabil, sondern verändern sich im Vollzug des Handelns (Giddens, 1988; Schimank, 2002). Unsere Aufmerksamkeit richtet sich dabei auf diese Veränderungen selbst, einerseits auf die Veränderung der Strategien und der Programmatik der einzelnen Akteure und andererseits auf den Wandel ihrer Positionen im bildungspolitischen Feld und auf dessen Neustrukturierung in neuen Akteurkonstellationen (Schimank, 1988, S. 620f.).

Der Bezugspunkt der von uns untersuchten Handlungsintentionen stellt immer das Bildungswesen und seine Gestaltung dar. Entscheidend ist dabei zu klären, in welchem Masse sich dieses von innen heraus, in einer Anpassungsleistung 
und eigener Dynamik ändert (Schimank, 2002, S. 173f.) oder auf externe Steuerungsimpulse reagiert. Die historische Sicht auf die Steuerungsvorstellungen bezüglich des Bildungswesens und die Wirksamkeit der unterschiedlichen Akteure soll erlauben, eine Vorstellung über die Ausdifferenzierung der Bildungspolitik selbst zu gewinnen.

Für die Analyse der Entwicklung des institutionellen Gefüges und der Verteilung der Entscheidungskompetenzen in der Bildungspolitik beziehen wir uns auf die Unterscheidung eines "dezentralen» von einem "unitarischen Föderalismus» (Braun, 2003). Während der unitarische Föderalismus als eine Form der Herbeiführung einheitlicher Entscheide und der Partizipation der Gliedstaaten beschrieben werden kann, wird der dezentrale Föderalismus als eine Machtverteilung verstanden, die geradezu darauf angelegt ist, Vereinheitlichung abzubremsen und Differenzierung zu erhalten.

\section{Das Ende des bildungspolitischen Sonderfalls?}

PISA als OECD-Projekt ist Teil eines Indikatorenprogramms, welches den Mitgliedstaaten periodisch vergleichende Daten zu Ressourcenausstattung und Leistungsfähigkeit ihrer Bildungssysteme zur Verfügung stellen will (Baumert et al., 2002, S. 11f.). Leitend ist die Vorstellung, dass international vergleichende Studien und die Ermittlung der «best practice» transnational kollektive Lernprozesse auszulösen vermögen und dadurch zur Verbesserung nationaler bildungspolitischer Steuerungs- und Allokationsprozesse beitragen können.

Was PISA und ähnliche frühere Studien wie TIMSS (Third International Mathematics and Science Study) und IALS (International Adult Literacy Survey) bildungspolitisch bedeuten, wird dann ersichtlich, wenn ihre Anlagen mit andern Evaluationspraktiken der OECD verglichen werden, wie sie dem Expertenbericht der OECD über die Bildungspolitik (1991), den Berichten über die Erwachsenenbildung (2001) und über das tertiäre Bildungssystem (2003) zugrunde lagen.

Charakteristisch für die traditionellen Examen sind folgende Merkmale (Expertenberichte der OECD zur Erwachsenenbildung, 2001, S. 1ff. und Expertenbericht der OECD zur Tertiären Bildung, 2003, S. 12ff.): Die Initiative wird von den Ländern ergriffen, die einen Bildungssektor evaluieren lassen wollen. Die thematischen Schwerpunkte und die Verfahren der Examen werden bilateral ausgehandelt. Daran sind bildungspolitisch legitimierte Akteure, Repräsentanten aus Politik und Verwaltung beteiligt. Thematisch standen bisher Struktur- und Entwicklungsfragen im Vordergrund. Die Evaluation basiert auf dem klassischen Muster: Selbstberichterstattung ("Länderbericht») und Treffen vor Ort, die vom zu evaluierenden Land organisiert werden. Die Gespräche werden von Seiten der OECD durch Gruppen aus Wissenschaftlern und bildungspolitischen Repräsentanten geführt, welche in andern Ländern rekrutiert werden. Ihre 
Resultate werden in einem Bericht ausgewertet, allenfalls mit zusätzlichen statistischen Analysen unterlegt. Sowohl der Länder-als auch der Expertenbericht der OECD werden nach Diskussionen mit Vertretern der Evaluierten selbst überarbeitet und erst dann verabschiedet.

In diesem Verfahren definieren die Evaluierten in erheblichen Masse Problemlagen mit, die weichen Erhebungsmethoden schaffen dazu einen Raum, welcher der eigenständigen Zielsetzung der Evaluierten genügend Platz lässt. Namentlich können die Befragten die Experten und Expertinnen auf spezifische Aspekte im Bildungswesen (z.B. Sonderfall Schweiz mit 26 Bildungssystemen) aufmerksam machen und auf deren gebührende Berücksichtigung hinwirken. ${ }^{3}$ Der internationale Bezug und Vergleich ist wesentlich im Wissen und in den Erfahrungen der Experten und Expertinnen repräsentiert, ohne dass dieser gegenüber den Anliegen der Evaluierten dominant wird (Evaluation Erwachsenenbildung: Adult Learning Policies and Practices, 2003).

Gegenüber diesen herkömmlichen Verfahren zeichnet sich PISA durch neue Qualitäten aus: Zunächst stellt das Verfahren aus der Sicht der OECD ein bildungspolitisches Steuerungsinstrument dar, das überall gleichermassen eingesetzt werden kann/soll. Dann wird das Projekt in einem Netzwerk aller beteiligten Länder geplant und realisiert. Dieses stellt eine relativ komplexe multilaterale Situation und Struktur dar, welche den Handlungsspielraum der Vertreter eines Landes erheblich begrenzt. Weiter fokussiert die Fragestellung die Analyse von Leistungen/Ergebnissen (Outcomes) des Bildungssystems und thematisiert dadurch vergleichend nationale Grundausstattungen mit Bildungsressourcen. Die Analyse wird zudem mit gemeinsam erarbeiteten standardisierten Verfahren durchgeführt, welche den herkömmlichen, wissenschaftlich definierten Anforderungen genügen müssen. Projektplanung, Erhebung, Analyse und Berichterstattung erfolgen somit weitgehend dekontextualisiert vom je lokal spezifischen Untersuchungsgegenstand und unterliegen einer fachwissenschaftlichen Kontrolle. Ergebnisse und deren Interpretation werden unter wissenschaftlichen, universalistischen Gesichtspunkten hervorgebracht bzw. vorgenommen, und nicht einzelnen Akteuren, den Anliegen der Evaluierten angepasst. Sie sind kaum mehr bildungspolitisch verhandelbar.

Anders steht es mit den Folgerungen, welche aus derartigen Studien abgeleitet werden. Ihre Interpretation bzw. ihre Verwendung wird weitgehend in den spezifischen nationalen Kontexten ausgehandelt. Schliesslich stellt sich das Ergebnis als eine Rangordnung dar, wobei der erste Platz die beste Erfüllung der definierten Zielgrössen repräsentiert und sichtbar macht.

Ganz in diesem Sinne hat auch die EDK, die Erziehungsdirektorenkonferenz, die traditionelle Hüterin der Vielfalt des schweizerischen Bildungswesens, in ihrer Erklärung vom 7. März 2002 die erwähnten Ergebnisse als nicht hintergehbare Fakten wahrgenommen (http://www.edk.ch/PDF_Downloads/Empfehlungen/Deutsch/Erkl_Pisa_d.pdf). Sie macht die Fremddiagnose zu ihrer eigenen. Zwar sollen die Ergebnisse der Studie im Jahre 2002 noch detailliert 
analysiert und interpretiert werden, aber sofort wird auch ganz allgemein ein "primärer Handlungsbedarf» bei der Förderung der Lesefähigkeit in Richtung der anerkannten Zielgrösse diagnostiziert (EDK, 2002, S. 2). Wenn sie dabei die «Schwerpunkte ihres aktuellen Tätigkeitsprogramms» bestätigt sieht, verweist sie selbst auf vereinheitlichende Steuerungsgrössen hin (EDK, 2002, S. 3) - wie die Standards in den Kernfachbereichen der obligatorischen Schule, die künftig zur Geltung gebracht werden sollen.

In Analogie zu diesem Verlauf kann die Bedeutung des so genannten Bolognaprozesses beschrieben werden. Während bislang die einzelnen Kantone und der Bund als Hochschulträger auf Autonomie und Vielfalt beharrten, sind es gegenwärtig nicht allein die institutionellen Akteure des Bundes, sondern auch jene der Koordination der einzelnen Hochschulen und der Hochschulkantone selbst, die sich für die Vereinheitlichung der Abschlüsse entsprechend dem europäischen Bologna-Modell einzusetzen beginnen.

\section{Zentralismus-Föderalismus: Transformation und überlagerung des konstitutiven bildungspoliti- schen Gegensatzes}

Im folgenden Abschnitt wird dargelegt, wie in der Bildungspolitik des modernen Bundesstaates, trotz dessen Festlegung auf dezentrale föderalistische Strukturen, der Gegensatz der föderalen zur zentralen Entscheidung politisch virulent geblieben ist. Zentrale, bundesstaatliche Entscheide werden dabei mit der Vereinheitlichung des Bildungswesens gleichgesetzt, während den föderalen Entscheiden die Erhaltung der Diversität kantonaler Bildungswesen und deren Koordination zugeordnet werden. Im ausgehenden 20. Jahrhundert wird dieser Gegensatz bzw. die Zuordnung abgelöst durch einen Prozess, der eine Vielzahl von institutionellen Akteuren auf Bundesebene und im Bereich der Koordination der Kantone profiliert, die sich zum Teil konkurrenzieren, aber gleichermassen vereinheitlichende bzw. koordinierende Lösungen favorisieren.

Der Politologe Dietmar Braun beschreibt das schweizerische politische System allgemein als «dezentralen Föderalismus» und profiliert diesen am deutschen «unitarischen Föderalismus» (Braun, 2003). Im dezentralen Föderalismus behalten die Gliedstaaten, die Kantone, ihre politische und kulturelle Autonomie, das Prinzip ihres Zusammenschlusses ist die Nicht-Vereinheitlichung. Dem zentralen Staat wurde historisch v.a. die Funktion des Schutzes der Demokratie nach aussen und innen und die Herstellung eines einheitlichen Wirtschaftsraumes zugemessen (Braun, 2003, S. 60). ${ }^{4}$

Den Kantonen kommt entsprechend dieser Unterscheidung auch nicht eine Vetorolle im politischen Entscheidungsprozesses im Sinne einer «divided government» zu (Braun, 2003, S. 69), sondern bei der Ausführung von Entscheiden, die auf Bundesebene gefällt werden, können sie in mehr oder weniger gros- 
ser Distanz zu den Absichten des Bundes ihre eigenen Ziele verfolgen; damit können weder Bund noch einzelne Kantone unilateral ihre Interessen durchsetzen (Braun, 2003, S. 74). Doch auch diese «vertikale Verflechtung» von Bund und Kantonen wird nach Braun zunehmend von einer «horizontalen Verflechtung», Koordinierung der Kantone unter sich, abgelöst, in der Wettbewerb durch Innovationen und konsensuale Solidarität gleichzeitig gewährleistet sind (Braun, 2003, S. 82).

Die Identität des dezentralen Föderalismus beruht nach Braun auf der Sicherstellung der Vielfalt. Dementsprechend «bedarf es der eindeutigen Dominanz eines Diskurses», des Vielfaltdiskurses gegenüber dem Vereinheitlichungsdiskurs (Braun, 2003, S. 86). Wer etwas vereinheitlichen will, brauche dafür eine besondere Legitimation, während die Erhaltung oder auch die Herstellung von Vielfalt schon immer als Notwendigkeit gegeben und keiner Legitimation bedürftig erachtet wird.

Auch wenn die Beschreibung des Bildungssystems der Schweiz, bzw. der 26 differenten Bildungssysteme seit Bestehen der modernen Schweiz, bestens Brauns Thesen über den dezentralen Föderalismus zu bestätigen scheint, so zeigt sich doch, dass im bildungspolitischen Selbstverständnis in der Schweiz dieser Sachverhalt, die Dominanz des Vielfaltdiskurses, historisch keineswegs als gegeben angenommen werden konnte und auch kontinuierlich in Frage gestellt wurde. Der Vielfaltdiskurs hat diesbezüglich gerade nicht die Hegemonie gewonnen.

\section{Die programmatische Konstitution der bildungspolitischen Vereinheitlichung}

Seit Bestehen des modernen Bundesstaates besteht und erhält sich ein starker pädagogischer Vereinheitlichungsdiskurs, und die Vereinheitlichung setzte sich in bestimmten Bereichen und historischen Phasen auch immer wieder durch.

Bereits im Vorfeld der Gründung des modernen Bundesstaates äusserte sich der für den modernen Liberalismus repräsentative Ludwig Snell dazu programmatisch. Gerade weil die Vielfalt der Schweiz gegeben und auch nach der Gründung eines neuen Bundesstaates bewahrt bleiben soll, brauche es «eine und dieselbe Idee der Volksschule» (Snell, 1840, S. 43), welche die Basisausbildung, den Zugang zum rationalen Wissen aller künftigen Bürger als Grundlage der plebiszitären Demokratie und der rechtlichen Gleichheit vereinheitliche. Die in der Erinnerung der modernen Liberalen als glücklos mehr oder weniger verdrängte Helvetische Einheitsrepublik wird gerade im pädagogischen Einheits-Programm und Zentralismus des damaligen Ministers Stapfer wieder zum Vorbild. Dieses Argument bleibt bis weit ins 20. Jahrhundert eine der Grundlagen dafür, dass auch in einem dezentralen Föderalismus bildungspolitisch immer wieder der Einheits- oder Vereinheitlichungsdiskurs in der Bildungspolitik Oberhand gewinnen konnte und auch regelmässig versucht wurde, die Bildungsinstitutionen $\mathrm{zu}$ vereinheitlichen und auch bildungspolitische Akteure sich um dieses Programm formierten. ${ }^{5}$ 
P. I. V. Troxler, der unter den schweizerischen Erneuerern das Konzept des vielfältigen Bundesstaates nach dem Beispiel der USA propagierte und dieses Konzept auch in die Verfassungsdiskussion von 1848 einbrachte (Troxler, 1848), gehörte Zeit seines Lebens zu den Befürwortern eines einheitlichen schweizerischen Bildungssystems und machte dazu auch schon sehr früh einen Vorschlag, der zeigen sollte, wie diese Vereinheitlichung institutionell erreicht werden konnte. Die «Gesamthochschule» bilde gewissermassen «die Krone der Nationalbildung» (Troxler, 1830, S. 163) und vereinheitliche von hier aus institutionell und inhaltlich die zuführenden kantonalen Bildungssysteme.

Die Vereinheitlichung der Bildung findet damit gerade angesichts des dezentralen Föderalismus eine pädagogische, d.h. eine juridische und eine institutionelle Begründung und wurde in der Folge immer wieder als Aufgabe der zentralen politischen Macht zugeteilt.

In der Verfassung von 1848 wurde die Errichtung einer Universität und einer polytechnischen Schule - nach damaliger Terminologie eine Form höherer Volksschule, die auf Technologie und Empirie ausgerichtet ist - durch den Bund vorgesehen. ${ }^{6}$ Auch wenn versichert wurde, dass die Schulen selbst in der Hoheit der Kantone bleiben sollten (Klöti, 1903, S. 2), ging man davon aus, dass die nationalen Institutionen regulierend und vereinheitlichend nach unten wirken würden.

\section{Kontinuität des Konflikts}

Der Ausführung dieser Verfassungsbestimmung - die schliesslich 1854/55 nur zur polytechnischen Schule, der künftigen ETH führte - war eine mehrere Jahre dauernde Auseinandersetzung gewidmet, die schliesslich prototypisch für die künftige Auseinandersetzung über die Optionen Zentralismus/Bildungsvereinheitlichung versus föderalistische Machtaufteilung/Vielfalt werden sollte (Winter, 1998, S. 380-385).

In der Revision der Bundesverfassung 1872/74 wurde die Frage nach dem Mass der bildungspolitischen Vereinheitlichung im Rahmen einer zentralstaatlichen Regelung zu einem der entscheidenden Zankäpfel und führte schliesslich zur Einführung der Kontrollkompetenz des Bundes über das Volksschulwesen (Criblez, 1999). Ganz unterschiedliche Akteure und Begründungen fanden sich dabei auf beiden Seiten der Auseinandersetzung (Aubert, 1967, S. 44ff.).

Als 1882 die entsprechende Gesetzgebungsarbeit und die Institutionalisierung der zentralen Vereinheitlichungsinstanz, das eidgenössische Schulsekretariat, im ersten Referendumskampf gestoppt wurde, waren beide Seiten erneut durch unterschiedlichste Kräfte und Beweggründe bestimmt. Im so genannten «Schulvogt-Kampf» standen sich nicht nur Zentralisten und Föderalisten, sondern ebenso diejenigen, die für oder gegen die Trennung von Kirche und Staat oder für oder gegen den Ausbau der demokratischen Rechte eintraten, gegenüber. Die professionspolitisch mächtige Vereinigung der Lehrer versuchte zudem über die Vereinheitlichung auch die Freizügigkeit in der Zulassung zur Berufs- 
ausübung zu erreichen (Fäh, 1903). Die Vereinheitlichung des Bildungswesens und die politische Zentralisierung wurden durch Akteure befördert, die in andern Bereichen zu den vehementen Befürwortern der Vielfalt und der dezentralen Koordinierung gehörten und umgekehrt (Hard, 1974, S. 157-160).

Trotz des Misserfolges konnten sich zentral gesteuerte Vereinheitlichungstendenzen an wichtigen Schnittstellen des Bildungswesens durchsetzen. Die eidgenössische Rekrutenprüfung wurde gezielt zur Vereinheitlichung der kantonalen Volksschulen eingesetzt. Nationale Ratings, nach Kanton und sogar Gemeinden aufgegliedert und landesweit veröffentlicht, erzeugten einen Druck auf die niederrangierten Kantone, ihre Curricula und Bildungsleistungen an die nationalen Standards anzupassen, um «ihre» Rekruten vor der Schmach zu bewahren - was die Steuerungswirkung eines Rating wie PISA im kleineren Rahmen belegt.

Die Regelung der Zulassung zu den Medizinal- und Veterinärsprüfungen von 1880, die Grundlage der Freizügigkeit der Berufsausübung im Gesundheitssektor (Braun, 1985) und die Zulassungsregelung zu den Studien an der ETH führten de facto zur Vereinheitlichung der Maturitätsprüfungen und damit der Gymnasien selbst (Surdez, 1998). Sie gab auch dem Bund im Rahmen der Akzeptanz der Vielfalt eine wirkungsvolle Möglichkeit, regelnd einzugreifen und die Mindestanforderung an die Maturität durch die Institution der EMK (Eidgenössische Maturitätskommission) ständig zu erneuern und deren Erfüllung durch die kantonalen Schulen zu überwachen (Barth, 1919).

Auf der andern Seite führte aber der Versuch des Bundes, über Subventionen erneut gegenüber den Volksschulen vereinheitlichend vorzugehen, 1897/98 zum Entstehen des Prototyps der «horizontalen Verflechtung» in der Schweizerischen Konferenz der kantonalen Erziehungsdirektoren (EDK). Mit diesem Begriff bezeichnet Braun die für den dezentralen Föderalismus typische, koordinierende und konsensuale Zusammenarbeit der Kantone zur Wahrung der Diversität (Braun, 2003, S. 79). Die Erziehungsdirektoren einigten sich auf einen Subventionsmodus, der de facto jegliche Einflussnahme des Bundes auf die Schulgestaltung verhinderte (alte BV Art. 27bis). Die feste Einrichtung der Erziehungsdirektorenkonferenz musste allerdings mit einem sehr niedrigen institutionellen Profil erfolgen, um zu verhindern, dass sie selbst zu einem Vereinheitlichungsfaktor wurde (Weisser, 1997, S. 65).

\section{Vereinheitlichung versus Vielfalt in der Expansions- phase}

Auch die Expansion des Bildungswesens in der zweiten Hälfte des 20. Jahrhunderts wurde nach wie vor durch die Rivalität der beiden Muster politische Zentralisierung/Vereinheitlichung und horizontale Verflechtung/Vielfalt des Bildungswesens geprägt, wobei allerdings auf beiden Seiten ein schneller institutioneller Ausbau von Regelungs- und Vereinheitlichungs- bzw. Koordinationsmechanismen vorangetrieben wurde. 
Die EDK schuf vier regionale Koordinationsräume und -gremien, die versuchen sollten, die Koordinationsanstrengungen zu intensivieren. 1970 wurde unter einstimmiger Zustimmung der Erziehungsdirektoren der Kantone das Konkordat über die Schulkoordination geschlossen, das zum ersten Mal Koordinationsvorgaben bindend enthielt. Nachdem aber in kantonalen Volksabstimmungen einzelne Regelungen im Namen der Vielfalt bekämpft und zurückgenommen wurden und sich der Inhalt der Vereinbarung nur über eine Regelung in der Bundesverfassung retten liess, konnte die Perspektive einer politischen Steuerung des schweizerischen Bildungswesens über ein koordinierendes Konsensorgan an der Öffentlichkeit kaum an politischer Glaubwürdigkeit gewinnen (Badertscher, 1997, S. 195, 216).

Doch auch die zentralistischen Regelungen konnten sich in der Expansion in der zweiten Hälfte des 20. Jahrhunderts nicht als erfolgversprechende Alternative bewähren. Die Berufsbildung, die seit 1930 durch den Bund geregelt wurde, entwickelte sich in der starken Expansion in der Nachkriegszeit zwar zum Muster dessen, was als "vertikale Verflechtung» gelten kann, in der dem Bund eine bedeutende Aufgabe zugeschrieben wird (Braun, 2003, S. 79).7 Auf Hochschulebene, wo mit dem Kadermangel im Wirtschaftsaufschwung der bildungspolitische Handlungsbedarf am brennendsten wahrgenommen wurde, schuf sich der Bund mit dem Wissenschaftsrat einen Wissensvorsprung, der ihm erlauben sollte, gegenüber den Kantonen eine vereinheitlichte schweizerische Hochschulund Forschungspolitik durchzusetzen. ${ }^{8}$ In dem Sinne wurde 1968 auch das Bundesgesetz über die Hochschulförderung erlassen, das dem Bund gestützt auf seine finanziellen Beiträge die Federführung in der Koordination bzw. Vereinheitlichung der kantonalen Hochschulen zu einem schweizerischen Hochschulraum übergab. Gemäss der Gesetzesformulierung sollte «der Bund namentlich auf die Zusammenarbeit aller Hochschulen wirken» und unter diesem Gesichtspunkt auch seine finanziellen Beiträge ausrichten (Art. 1, Abs. $1 \& 2$ ). ${ }^{9}$

Diese Versuche einer vertikalen Verflechtung der Universitäts- und Wissenschaftspolitik unter zentraler Führung mit vereinheitlichenden Perspektiven wurden 1978 durch ein siegreiches Referendum des Schweizerischen Gewerbeverbandes gegen die Revision des Hochschulförderungsgesetzes empfindlich behindert. Der Ausgleich der Hochschulkosten zwischen den Kantonen musste schliesslich durch ein Konkordat ohne Steuerungsmöglichkeiten geregelt werden (Meier, 1983). Auch der Versuch des Bundes, auf die Blockierung der EDK im Konkordat mit der Schaffung von Bundeskompetenzen zu reagieren und das nachobligatorische Bildungswesen der Rahmenregelung des Bundes zu unterstellen, scheiterte 1973 in der Volksabstimmung am Ständemehr.

Der Konfliktherd zwischen zentralstaatlichen, mehr oder weniger vereinheitlichenden Regelungen und den koordinierenden, horizontalen Verflechtungen der Kantone zur Wahrung der Diversität war weitgehend aktiv in der Bildungspolitik bis in die Expansionsphase um 1970, dabei verloren aber beide Perspektiven mehr oder weniger ihre Durchschlagskraft. 
Neue institutionelle Akteure in der Bildungspolitik und Ausbau institutioneller Akteure

Allerdings bedeutete dies keineswegs eine Lähmung der schweizerischen Bildungspolitik, vielmehr wird deren Gravitationszentrum zunehmend aus dem traditionellen Spannungsfeld herausgerückt. Basis für diese Entwicklung ist die verstärkte Institutionenbildung und -differenzierung im Bereich der Bildungsverwaltung, sowohl auf der Seite des Bundes als auch auf der Seite der Kantone und schliesslich auch der traditionellen Koordinationsstellen.

Auf der Seite des Bundes tritt das Bundesamt für Berufsbildung und Technik (BBT) nicht mehr nur in seiner Rolle als Koordinationsorgan der Ausführung des Berufsbildungsgesetzes durch die Kantone auf, mit der Bundesregelung der Fachhochschulen wächst ihm auch eine wichtige Aufgabe im Bereich des tertiären und mit der Berufsmatur im sekundären Bildungswesens zu. Neben den Wissenschaftsrat, der den Bundesrat berät, und das traditionelle Bundesamt für Bildung und Wissenschaft im Departement des Innern, tritt im gleichen Ministerium die Gruppe für Wissenschaft und Forschung (GWF), die beansprucht, verwaltungsmässig alle Anstrengungen der Wissenschafts-, Forschungs- und Hochschulpolitik zu bündeln und national und international $\mathrm{zu}$ artikulieren (GWF, 2003). ${ }^{10}$

Das Bundesamt für Statistik wurde im Bereich der Bildungsstatistik wesentlich ausgebaut und kann heute einen ganzen Set von Massstäben vorlegen, an denen sich die Steuerung auszurichten hat.

Auch auf der Ebene der Kantone treten zunehmend ausdifferenzierte Verwaltungseinheiten auf, die Bildungspolitik nicht nur planend vorbereiten, sondern auch ausführend begleiten und eigene Steuerungsinstrumente dafür entwickeln (Kussau \& Oertel, 2001). ${ }^{11}$

Selbst die traditionellen Organe der horizontalen, koordinierenden Verflechtung entwickeln eine Tätigkeit, die v.a. auf dem institutionellen Ausbau von eigenständigen Verwaltungseinheiten mit administrativer oder planend-wissenschaftlicher Zielsetzung beruhen. An erster Stelle steht hier ohne Zweifel die EDK, die nach einem sukzessiven Ausbau sich 1995 unter dem Stichwort, von der Schulkoordination zu einer umfassenden Bildungspolitik' reorganisiert hat und sich neben den regionalen Abteilungen Kommissionen für die Fachhochschulen, die Berufsbildung und die Erwachsenenbildung schuf, die mit wissenschaftlichen und administrativen Stäben national und international agieren (Arnet, 1997, S. 228-241) und dabei selbst vereinheitlichende und verbindliche Lösungen vorbereiten und vorantreiben.

An der Seite der EDK geben sich aber auch andere traditionelle Koordinationsorgane, wie die Schweizerische Hochschulrektorenkonferenz, jetzt Rektorenkonferenz der Schweizer Universitäten (CRUS), und ihr politisches Pendant, das Koordinationsorgan der Hochschul-Kantone, ${ }^{12}$ die Schweizerische Universitätskonferenz (SUK), zunehmend stärker werdende eigene Stäbe für «die strategische Mehrjahresplanung für den Bereich der universitären Hochschulen» 
(CRUS, 2003). Auf der Ebene der Fachhochschulen findet diese Entwicklung eine genaue Entsprechung.

\section{Ausdifferenzierung des bildungspolitischen Feldes}

Diese institutionelle Differenzierung in der Bildungspolitik und die Vervielfachung von Verwaltungseinheiten, die alle ein eigenes Tätigkeitsfeld bearbeiten, führt auch zu einer Neustrukturierung des Feldes. Neben den traditionellen Bereich der politischen Entscheide, woran die horizontalen und vertikalen Verflechtungen der Ausführung anschlossen, tritt für diese Verwaltungseinheiten das Feld der eigentlichen Bildungseinrichtungen, das nach Vorstellungen von Management und strategischer Führung strukturiert und bearbeitet wird. Das dritte Feld bildet die verwaltungsnahe Bildungsforschung, der dabei die Funktion zukommt, Steuerungswissen zu produzieren und zirkulieren zu lassen. Charakteristisch ist jedoch, dass diese drei Felder teilweise überlappen, deren Akteure also nicht ganz autonom agieren können (Ladwig, 1994, S. 345).

\section{Verschiedene Vereinheitlichungsprozesse ohne Zentralisierung}

In dieser Perspektive verschwindet das traditionelle Spannungsfeld von bundesstaatlicher und dezentraler Regelung nicht einfach, aber die Perspektive der Vereinheitlichung oder der Koordination wird zunehmend davon abgekoppelt. Auch zentrale Regelungen setzen weitgehend auf Koordination und umgekehrt betreiben die damit immer gewichtiger platzierten und zahlreicher in Erscheinung tretenden Organe und Stäbe der horizontalen Verflechtung, die ursprünglich die Diversität durch Koordination schützten, ebenfalls eine vereinheitlichende Politik. Schematisch kann diese Überlagerung und ihre Problematik an drei besonders bedeutungsvollen Beispielen gezeigt werden. Mit dem Fachhochschulgesetz überliessen die Kantone dem Bund den stark expandierenden Bereich der Fachhochschulen zur Regelung, ohne dass dies eine Zentralismus-Auseinandersetzung auslöste und auch ohne dass unzählige Sicherungen für die Garantie der Diversität eingebaut wurden. Aber auch der umgekehrte Fall erzeugt die gleiche Attraktivität. Historisch war die Auseinandersetzung über die Maturitätsanerkennung besonders beladen durch den Gegensatz von Bund und Kantonen. Schon früh verstand es der Bund, auf diesem Feld eine einheitliche Lösung gegen die Vielfalt der kantonalen Lösungen und deren Koordination durchzusetzen. Im Hinblick auf die Revision der Maturitätsanerkennungsverordnung beschloss die EDK 1993 einen Rahmenlehrplan für die gymnasialen Maturitätsschulen, um dann 1995 sogar die Einheitslösung neben der Bundesverordnung in einem komplizierten juristischen Verfahren zu einem für alle Kantone verbindlichen Reglement zu machen (Meylan, 1997, S. 58). Die Vereinheitlichung der universitären Studiengänge nach dem Bolognamodell ist nicht gebunden an die Initiative des Bundes, sondern wird auch vorangetrieben durch die traditionellen Organe der kantonalen horizontalen Politikverflechtung wie CRUS und SUK. 
Dabei baut sich allerdings ein ganz neuer Spannungstypus auf. Die Vereinheitlichung der Matur, die Einführung der Berufsmatur, die Regelung der Fachhochschulen und die Vereinheitlichung der universitären Studiengänge sind unter sich kaum aufeinander abgestimmt. Die Spannung verläuft dabei nicht zwischen den Akteuren von Bund und Kantonen, es wird nicht programmatisch gewählt zwischen Vereinheitlichung und Diversität, sondern für jeden Bereich bildet sich eine spezifische Akteurkonstellation heraus, die eine eigene Strategie für den jeweiligen Bereich entwickelt, ohne dass diese aufeinander abgestimmt sind.

Programmatisch treten sowohl das Konzept der Vielfalt, wodurch das Bildungswesen auf die kulturellen Unterschiede und Selbständigkeit im Föderalismus ausgerichtet wird, wie auch jenes der Vereinheitlichung zur Alimentierung von Öffentlichkeit und Wohlfahrt durch Bildung zunehmend in den Hintergrund.

\section{Veränderter internationaler Kontext}

Im Folgenden wird diskutiert, wie sich das internationale bildungspolitische Umfeld der Schweiz in den letzten Jahren verändert hat und welcher Wandel in der bildungspolitischen Programmatik damit verbunden ist. In unserem Blick stehen jene Akteure, denen wir mit einer gewissen Plausibilität eine unmittelbare Relevanz für die Gestaltung des schweizerischen Bildungssystems zuschreiben können. ${ }^{13}$

In der europäischen bildungspolitischen Landschaft stehen historisch lange der Europarat und die Europäische Union im Vordergrund. Der Europarat verstand sich als Antwort auf die katastrophale Situation, welche der Zweite Weltkrieg in Europa hinterlassen hatte (http://www.coe.int/). Am 5. Mai 1949 haben zehn europäische Länder im Londoner Vertrag die Bildung des Europarates beschlossen. Dadurch wurden die Voraussetzungen geschaffen, damit der Europarat als zwischenstaatliche Organisation, dessen Mitgliederzahl stetig erweitert wurde (neuerdings in Osteuropa), seine Aktivitäten zur Förderung der Demokratie, der Rechtsstaatlichkeit, der Menschenrechte wie auch der kulturellen Entwicklung und Identität in Europa entfalten konnte.

Die Programmatik ist kultur- und demokratiepolitisch ausgeprägt. Fragen wie die der staatsbürgerlichen Erziehung zur Demokratie, der europäischen Geschichte, der Sprachen, der Hochschulbildung und Forschung, der Mobilität und Anerkennung von Diplomen stellen vorrangige Themen dar. So kam auf Initiative des Europarates und der UNESCO 1997 die sogenannte Lissabonner Konvention zu Stande, welche von der Schweiz zwei Jahre später ratifiziert wurde. Diese Konvention regt insbesondere die Anerkennung von Qualifikationen und Abschlüssen beim Zugang zu den Hochschulstudien (Cottier, BreinigKaufmann \& Kennett, 2003, S. 65ff.) an. 
Auf programmatischer Ebene operiert komplementär zum Europarat die Europäische Union (http://europa.eu.int/). Die Hauptreferenz ihrer Programmatik muss im wirtschaftlich-strukturellen und seit den späteren 80er Jahren auch wohlfahrtsstaatlichen («social cohesion») Bereich gesehen werden. Seit den Römer Verträgen von 1957 hat in der EWG bzw. der EU die Bildungspolitik, insbesondere die Berufsbildungspolitik einen hohen Stellenwert (Field, 1998 und Müller-Solger, 1997). Ausgehend von Grundsätzen für die Berufsbildungspolitik in den 60er Jahren, über die Förderung der bilateralen Zusammenarbeit in den 70er Jahren, über die Durchführung der Bildungsprogramme (Erasmus, Sokrates usw.) führte die EU zu einer stetigen Verfestigung der Bildungszusammenarbeit unter den Mitgliedsländern. Diese konnte mit den Maastrichter Beschlüssen von 1993 konsolidiert werden.

Mit diesen Beschlüssen verfügt die EU über neue rechtliche Voraussetzungen, bildungspolitisch zu handeln. Der Vertrag überträgt der EU den Auftrag zu einer aktiven Berufsbildungspolitik: «Die Gemeinschaft führt eine Politik der beruflichen Bildung, welche die Massnahmen der Mitgliedstaaten unter strikter Beachtung der Verantwortung der Mitgliedstaaten für Inhalt und Gestaltung ergänzt.» Dabei geht es namentlich um «die Erleichterung der Anpassung an den industriellen Wandlungsprozess, insbesondere durch berufliche Bildung und Umschulung [...]» (Art. 127 EGV). Aktiv und komplementär soll die Berufsbildungspolitik, «subsidiärer» die allgemeine Bildungspolitik der EU gestaltet werden. Sie «trägt zur Entwicklung einer qualitativ hochstehenden Bildung bei [...] und fördert die Zusammenarbeit der Mitgliedstaaten [...] und unterstützt diese gegebenenfalls [...]» Im Vordergrund stehen hier u.a. die Entwicklung der europäischen Dimension im Bildungswesen (Sprachen), die Förderung der Mobilität der Lernenden (Art. 126 EGV).

Bezüglich dieser zurückhaltenden Politik setzte die EU 2000 mit der Umsetzung der Lissabonner Bildungsbeschlüsse der Staats- und Regierungschefs einen markanten Meilenstein für eine gemeinsame Bildungspolitik. Diese nutzen vier Methoden für die Koordination: Die Definition von Benchmarks; den Einsatz von Indikatoren, die gemeinsam festgelegt werden und dazu dienen, den Stand des Erfolges der eingeleiteten Massnahmen zu überprüfen; den Arbeitsaustausch über bewährte Praktiken und gemeinsame Projekte sowie die Durchführung von Peerreviews. Namentlich legen die Staats- und Regierungschefs für verschiedene Bereiche Zielvorgaben für 2010 fest und definieren die entsprechenden Messgrössen (Indikatoren) (Fabian, 2002, S. 127ff.). Dieses Verfahren verdeutlicht, dass die EU die Weiterentwicklung des Bildungsbereichs ihrer Mitgliedsländer in erster Linie über Transparenz und Wettbewerb und nicht so sehr über politische Massnahmen steuern will. Das Setzen von Benchmarks soll dabei offenbar harmonisierend wirken. Während die traditionelle Projektförderung mindestens zum Teil strukturunabhängig war, steht nun bewusst die Struktur und die Entwicklung des Systems im Blick des bildungspolitischen Handelns. 
Anders als die EU versteht sich die OECD (www.oecd.org/about vom 12.9.2003), die 30 Länder vereinigt, als Forum und Dienstleistungsanbieterin mit Beratungsfunktionen für die Regierungen. Aus dem Marshallplan nach dem Zweiten Weltkrieg herausgewachsen, operierte die OECD schon sehr früh mit Analysen, Entscheiden, Empfehlungen und Umsetzungsstrategien. Dialog und Konsensus haben in diesen Prozessen einen hohen Stellenwert. Der Bildungssektor ist seit jeher ein wichtiger Tätigkeitsbereich der OECD und in einer Reihe von Bildungsfragen kommt ihr seit den 50er Jahren eine wichtige Rolle zu bei der Definition von Handlungsfeldern und sogar -strategien der Mitgliedsländer. Dabei wirkte die internationale Organisation als Forum, über das bildungspolitische Strategien entworfen und ihre Implementierung und Vereinheitlichung informell vorbereitet werden konnten und von wo aus die nationalen Akteure mit Perspektiven ausgerüstet wurden. Für die OECD stellt Bildung eine wichtige individuelle und kollektive Ressource dar. Bildung des Einzelnen wird verstanden als Möglichkeit, sich zu entwickeln und die Beschäftigungsfähigkeit zu sichern. Für die Initiativen der OECD im Bildungsbereich bezeichnend sind seit jeher Empfehlungen und Länderstudien und neuerdings die Erarbeitung von Steuerungsgrössen wie TIMS und PISA, wie im zweiten Abschnitt dargestellt wurde. In den vergangenen Jahren wurde die OECD zunehmend zum zentralen bildungspolitischen internationalen Forum der entwickelten Länder. Entscheidende Impulse, Konzepte und auch Verfahren werden hier entwickelt und über eine vielfältige publizistische und Kurs-Praxis in die Mitgliedsländer diffundiert.

Abschliessend sei auch auf die relativ junge, seit 1995 bestehende WTO (World Trade Organization: http://www.wto.org/) bzw. auf GATS (General Agreement of Trades and Services: http://www.wto.org/english/tratop_e/serv_e/ gats_factfiction_e.htm) hingewiesen. Die WTO stellt die eigentliche Nachfolgeorganisation von GATT dar. Als einzige internationale Organisation befasst sie sich mit den globalen Regeln des weltweiten Handels. Die WTO-Vereinbarungen wollen vorrangig den freien Handel sicherstellen und dadurch zur Förderung der Wohlfahrt unter den beteiligten Ländern beitragen. Nach Auffassung der WTO sollen auch Bildungsdienstleistungen dem freien Handel zugänglich gemacht werden. Die Regeln der WTO werden von den 130 Mitgliedern konsensuell erarbeitet und von nationalen Parlamenten ratifiziert.

Wichtigstes Anliegen im Bildungsbereich ist die Liberalisierung des Zugangs zur Erbringungen von Dienstleistungen in der höheren und der ErwachsenenBildung. Dabei sollen gemäss GATS v.a. die grenzüberschreitende Erbringung von Bildungsdienstleistungen (z.B. e-Learning über Internet), ihre Nutzung im Ausland (z.B. für Studierende aus dem Ausland) sowie die kommerzielle Präsenz von Bildungsanbietern (z.B. ausländische Sprachinstitute) im Bereich der höheren Bildung und die Präsenz natürlicher Personen (ausländische Dozenten) in der Erwachsenenbildung ermöglicht werden (Scherrer, 2003, S. 60f.).

Auch wenn der GATS-Prozess noch nicht zu verbindlichen Vereinbarungen mit der EU - geführt hat, so ist jedoch erkennbar, dass er in Europa zu einer wei- 
teren Liberalisierung des Bildungsraumes führen wird und dabei die Einführung von ECTS (European Credit Transfer System) und den Bolognaprozess flankiert (Scherrer, 2003, S. 65). Im GATS-Prozess soll eine «funktionelle Integrationsstrategie» umgesetzt werden, indem die Bildungsanbieter sich gezielt um Kunden und eine zahlungskräftige Nachfrage bewerben können (ebd.). Bei einer solchen Entwicklung wäre im Falle der Schweiz der Bund verpflichtet, ausländische Hochschulen gleich zu subventionieren wie die schweizerischen, sofern jene die Anerkennungs- und Akkreditierungskriterien erfüllen (Cottier et al., 2003, S. 83).

Zusammenfassend können wir festhalten, dass auch im internationalen bildungspolitischen Umfeld der Schweiz sich die Zahl der kollektiven Akteure vermehrt hat. Wenn sich auch die Programmatik nicht wesentlich verändert hat, so ist aber doch die Akzentuierung eindeutig neu gesetzt worden. Während der Europarat noch ausgeprägt die Bildungspolitik auf demokratiepolitische und rechtspolitische Werte hin formulierte, gewinnt die Orientierung an wirtschaftspolitischen Ziel- und Rahmensetzung an Bedeutung. Das transnationale bildungspolitische Feld wird damit zunehmend bezüglich lokaler kultureller und politischer Verhältnisse dekontextualisiert und - grossräumiger - rekontextualisiert.

Mit diesem Wandel veränderten sich die Vorstellungen über die Rolle und das Ausmass der Interventionen der transnationalen Akteure bei der Transformation des Bildungswesens: Während für den Europarat die Veränderung des Bildungswesens weiterhin eine traditionelle nationale Aufgabe darstellt - eine Vorstellung welche die EU bis Maastricht teilte, und auch die OECD beschränkte sich traditionell auf Empfehlungen-, beanspruchen die übrigen Akteure stärker, in die Struktur der Bildungswesen einzugreifen: Sei dies über das systematische Anlegen vergleichender, wissenschaftlich gestützter Massstäbe oder sei es durch die Erzeugung eines realen Wettbewerbs, insbesondere im Bereich der höheren Bildung.

Mit der strukturellen und programmatischen Transnationalisierung der Bildungspolitik verliert gleichzeitig der nationale Staat ein Stück weit seine Kontrollfunktion über das Bildungswesens. Teils wird er ersetzt durch professionelle EvaluatorInnen, teils soll der Markt diese qualitätssichernde Funktion übernehmen. Qualität und Zielsetzung und Programm werden damit zunehmend an international definierten Präferenzen orientiert (Fuchs, 2003, S. 170f.).

\section{Schluss: Formen der Internationalisierung der föderalistischen Bildungspolitik}

Abschliessend wird diskutiert, wie der internationale Diskurs und die bildungspolitische Programmatik in der Schweiz wirksam werden und ob sich bereits heute Folgen dieser Entwicklung im Bildungswesen beobachten lassen.

Unübersehbar hat sich in den letzten Jahren die Konstellation der bildungspolitischen Akteure verändert. Transnationale Akteure treten neu offen im bil- 
dungspolitischen Feld auf, die Referenz auf sie und auf ihre Strategien wird zur Legitimation bildungspolitischer Massnahmen in der Schweiz. Zahlreiche Hinweise sprechen dafür, dass sich die nationale und die internationalen bildungspolitischen Arenen über Informationskanäle, Strukturbildungen, Vernetzungen und Personen - Entscheid und Experten - in Politik und Wissenschaft zunehmend verflechten. Wenn die Vereinheitlichungsstrategie in der Schweiz sich weitgehend vom Bund abgelöst hat und auch in Formen der horizontalen Verflechtung von Kantonen wie EDK oder SUK und Bildungsinstitutionen wie CRUS sich artikuliert, so findet sie in diesen transnationalen Akteuren und Strategien ihren zunehmend wirkungsmächtigeren Bezug. Dezentral orientierte und zentralisierende Akteure beteiligen sich gleichermassen an der Internationalisierung der Bildungspolitik und ihren Foren und richten ihre Politik auf die von dort ausgehenden Impulse aus. Während jahrzehntelang die Frage der Vereinheitlichung der Hochschulen unter der Aegide von zentralen Akteuren ein Zankapfel war, richten sich heute alle Hochschulen selbst, die horizontalen Koordinations-Akteure wie auch die zentralisierenden Akteure auf die gleiche internationale «Bologna»-Strategie aus. Während die EDK zur Abwehr einer Vereinheitlichung der Volksschulen unter der Aegide des Bundes entstand, betreibt sie heute selbst diese Vereinheitlichung durch die Ausrichtung an internationalen Standards.

In dem Sinne kann davon ausgegangen werden, dass die Internationalisierung der Bildungspolitik gewissermassen den konstitutiven Gegensatz der nationalen Bildungspolitik zwischen Vereinheitlichung und Aufrechterhaltung der Differenz abschwächt oder beseitigt. Ob sich in diesem Prozess die nationale Bildungspolitik, ihre Konstellationen und Akteure in den internationalen Rahmen integrieren und auflösen oder sich erst nationalstaatlich konstituieren, kann in dieser Phase der Entwicklung stichhaltig noch kaum belegt werden.

Zweitens fördert die Verflechtung von nationalen und internationalen bildungspolitischen Arenen sowohl selbstadaptive Prozesse in den Bildungssystemen als auch die Homogenisierung der Programmatik im nationalen Raum. ${ }^{14}$ Wissen wird in diesem Prozess zu einer zentralen Steuerungsressource, welche die Akteure je nach Handlungsspielraum selektiv nutzen (Weber, 2000, S. 208f.). So weisen zahlreiche Beobachtungen darauf hin, dass in der Bildungspolitik das Ausland als Referenz weiter zu Lasten kantonaler und schweizerischer Bezüge an Gewicht gewonnen hat. Dieser Referenzwandel ist für die Bildungspolitik bedeutungsvoll: Kulturelle und demokratiepolitische Bezüge regionaler und nationaler Bildungspolitik werden weniger wichtig, hingegen gewinnen die ökonomischen - zugleich transnationalen - an Bedeutung. Bildungspolitisches Handeln kann sich nun auf eine breitere, internationale Legitimationsbasis stützen, während das herkömmliche nationale oder kantonale Legitimationsmuster weiterhin je nach Situation, passender Problemlage und Intentionen der Akteure verwendet wird.

Drittens scheint die besondere Legitimität der ausländischen Referenz darauf zu beruhen, dass Bildungspolitik und das Bildungswesen der Schweiz einem sys- 
tematischen und wissenschaftlich inspirierten internationalen Vergleich und Wettbewerb ausgesetzt sind. Ständig werden ihre Strukturen und Leistungen mit denjenigen anderer Länder verglichen. Diese Vergleiche halten das Bewusstsein für das eigene Ungenügen und die eigenen Schwächen wach.

Schliesslich erzeugen und verstärken der programmatische Wandel des internationalen bildungspolitischen Diskurses, die Ausarbeitung, Verfeinerung und Nutzung bildungspolitischer Analyse- und Interventionsinstrumentarien bei den schweizerischen Akteuren die Vorstellungen und das Bedürfnis, das Bildungswesen politisch zu steuern. Die Instrumentarien dazu sind legitimiert von den Experten-Akteuren, welche das Bildungswesen, seine Stärken und Schwächen von aussen analysieren, beurteilen und entsprechende Problemlagen definieren. Experten und Expertinnen werden zu treibenden Kräften der Veränderung.

\section{Anmerkungen}

1 In der PISA-Studie wurde die Leistungsfähigkeit der 15-jährigen Schüler und Schülerinnen in den Bereichen Lesekompetenz, Mathematik und Naturwissenschaften erfasst. Das Instrument für die standardisierte Leistungsmessung wurde von den 32 beteiligten Ländern gemeinsam entwickelt. Die schweizerischen Resultate sind bekannt: In Mathematik erreichten die Schüler und Schülerinnen Spitzenwerte, in den beiden andern Bereichen dagegen landeten sie bestenfalls im Mittelfeld.

2 Nicht allein die Schweiz hat das Fremdurteil in hohem Masse akzeptiert. Andere Länder wie etwa Deutschland (Fuchs, 2003, S. 163f.) taten dasselbe. Nur ist dies für Deutschland nicht so überraschend. Hier hat der Föderalismus einen unitarischen Charakter, in der Schweiz dagegen einen dezentralen.

3 Dieses Verfahren birgt Risiken in sich. Heidenheimer (1996, S. 608) weist in seiner Studie darauf hin, dass es bei der Ausarbeitung und Genehmigung der ersten OECD-Studie zur schweizerischen Bildungspolitik in erheblichem Masse zu unterschiedlichen Problemwahrnehmungen gekommen sei. Solche Unterschiede hätten nicht nur bei den externen Experten beobachtet werden können, sondern auch zwischen den Experten und schweizerischen Behörden. In der Folge verzögerte sich das Beurteilungsverfahren des Berichtes erheblich.

4 Demgegenüber ist der unitarische Föderalismus historisch auf vereinheitlichte Lebensverhältnisse und Teilhabe und Kontrolle der Gliedstaaten im Vereinheitlichungsprozess ausgerichtet.

5 Die einheitliche öffentliche Bildung gilt seit den grossen liberalen Bildungsprojekten der Französischen Revolution als eine Grundvoraussetzung moderner plebiszitärer Demokratie. Der schweizerische Liberalismus folgt dabei sowohl doktrinär wie auch in der Gesetzgebung fast sklavisch dem Vorbild, für das Condorcet federführend war. Stapfer und Snell sind dabei repräsentativ für die jeweilige Periode, aber keineswegs einmalig (Osterwalder, 2000).

6 Zur Stellung dieser Konstruktion innerhalb der ersten Bundesverfassung siehe Aubert (1967, S. 35).

7 Gegenüber der Vielfalt der Berufe und den unterschiedlichen Interessen der Akteure aus Wirtschaft und Gewerbe war es nicht die Koordination der Kantone, sondern einzig und allein der Bund, der ein minimales Durchsetzungsvermögen hatte.

8 Auch die Errichtung des Schweizerischen Nationalfonds zur Förderung der wissenschaftlichen Forschung als Stiftung 1953 kann als ein Mittel, mit dem der Bund politisch direkt 
in die kantonalen Hochschulen vereinheitlichend eingreifen konnte, betrachtet werden. Inwiefern die Bundes-Regelung der Mittelzufuhr an die Stiftung allerdings Steuerungseffekte im Ausbau der Hochschulen erzeugte, kann hier nicht beurteilt werden.

9 Dass der Bund in der Folge diese Kompetenz kaum wahrgenommen hat, oder an deren Wahrnehmung von andern Akteuren wirkungsvoll gehindert wurde, sei hier nicht weiter ausgeführt. Immerhin verweist diese gesetzliche Regelung aber auf heutigen Bestrebungen vorgelagerte Projekte in die Richtung einer ,Hochschule Schweiz'.

10 Auch hier muss darauf hingewiesen werden, dass es sich dabei um eine Intention oder eine Programmatik eines institutionellen Akteurs handelt und nicht um die Beschreibung einer Wirkung.

11 Einige dieser relativ unabhängigen Dienststellen wurden inzwischen in Direktionsstäbe transformiert (z.B. in den Kantonen Bern und Zürich).

12 Der Bund arbeitet ohne Führungsanspruch als Hochschulträger u.a. in der SUK mit.

13 Allerdings werden wir die seit den 50er Jahren in bi- oder multilateralen Kontexten eingeleiteten Bemühungen zur arbeitsmarktbezogenen oder akademischen Anerkennung von Diplomen im Folgenden ausblenden (Baumeler, 2001).

14 Hier ist anzumerken, dass selbstverständlich nicht alle Bildungsbereiche gleichermassen einem Anpassungsdruck ausgesetzt sind: Hochschulen und Universitäten binden sich stärker in ein transnationales System ein als Schulen auf der Sekundarstufe 2 zum Beispiel. Dies mag darauf zurückzuführen sein, dass die Hochschulen allgemein weniger kontextspezifische Merkmale aufweisen als die Einrichtungen der übrigen Bildungsbereiche (Fowler, 1994, S. 94f.) Die Ausgestaltung eines gemeinsamen europäischen Hochschulraumes, den 29 europäische Minister 1999 in Bologna beschlossen haben, stellt in diesem Zusammenhang ein zwar herausragendes, aber nicht ganz überraschendes Ereignis mit allerdings nicht absehbaren Folgen dar.

\section{Literatur}

Arnet, M. (1997). Von den Aufgaben und den Kompetenzen der EDK und von deren Organisation. In H. Badertscher (Hrsg.), Die Schweizerische Konferenz der kantonalen Erziehungsdirektoren 1897-1997 (S. 228-236). Bern: Haupt.

Aubert, J.-F. (1967). Traité de droit constitutionnel Suisse. Vol I. Neuchâtel: Ides et Calendes.

Badertscher, H. (1997). Die EDK als Instrument der Kooperation im Bildungsföderalismus seit 1968. In H. Badertscher (Hrsg.), Die Schweizerische Konferenz der kantonalen Erziehungsdirektoren 1897-1997 (S. 173-227). Bern: Haupt.

Barth, A. (1919). Die Reform der höhern Mittelschulen der Schweiz. Untersuchungen und Vorschläge über die Maturitätsverhältnisse und andere Mittelschulfragen. Basel: Spittlers.

Baumeler, T. (2001). Anerkennung von schweizerischen Diplomen. Manus/Kursunterlagen. Bern: BBT.

Baumert, J., Artelt, C., Klieme, E., Neubrand, M., Prenzel, M., Schiefele, U., Schneider, W., Tillmann, K.-J. \& Weiss, M. (2002). PISA 2000 - Die Länder der Bundesrepublik Deutschland im Vergleich. Opladen: Leske + Budrich.

Braun, D. (2003). Dezentraler und unitarischer Föderalismus. Die Schweiz und Deutschland im Vergleich. Schweizerische Zeitschrift für Politikwissenschaft, (1), 57-90.

Braun, R. (1985). Die Professionalisierung des Ärztestandes in der Schweiz. In W. Conze \& J. Kocka (Hrsg.), Bildungsbürgertum im 19. Jahrhundert. Bildungssystem und Professionalisierung in internationalen Vergleichen (S. 332-357). Stuttgart: Klett.

Cottier, Th., Breining-Kaufmann, Ch. \& Kennett, M. (2003). Liberalisation of Higher Education Services in Switzerland. The Impact of the General Agreement on Trade in Services (GATS). In Bundesamt für Bildung und Wissenschaft (Hrsg.), Die Auswirkungen des GATS auf das Bildungssystem der Schweiz (S. 65-147). Bern: BBW. 
Criblez, L. (1999). Der Bildungsartikel in der Bundesverfassung vom 29. Mai 1874. In L. Criblez et al. (Hrsg.), Eine Schule für die Demokratie (S. 337-362). Bern: Lang.

CRUS: Homepage. http://www.crus.ch/deutsch/CRUS/kurz.html, 1.10.2003.

EDK (2003). Erklärung der EDK zu den Ergebnissen von «PISA 2000». http://www.edk.ch/PDF_Downloads/Empfehlungen/Deutsch/Erkl_Pisa_d.pdf, 10.10.2003.

Europäische Union: Homepage. http://europa.eu.int/, 10.10.2003.

Europarat: Homepage. http://www.coe.int/, 10.10.2003.

Fabian, B. (2002). EUROSTAT. Europäische Bildungs- und Beschäftigungsstatistiken im Aufwind. Grundlagen der Weiterbildung, 187-286.

Fäh, F. (1903). Zur Freizügigkeit der Lehrer in der deutschen Schweiz. Die Geschichte des Konkordats-Projekts aus den Jahren 1881-83. Schweizerische pädagogische Zeitschrift, 13, 57-72.

Field, J. (1998). European Dimensions. Education, Training, and the European Union. London: Kingsley.

Fowler, F. C. (1995). The Neoliberal Value Shift and its Implications for Federal Education Policy under Clinton. Educational Administration Quarterly, 31(1), 38-60.

Fuchs, H.-W. (2003). Auf dem Weg zu einem Weltcurriculum? Zeitschrift für Pädagogik, 161179.

GATS: Homepage. http://www.wto.org/english/tratop_e/serv_e/gats_factfiction_e.htm, 10.10.2003.

Giddens, A. (1988). Die Konstitution der Gesellschaft. Frankfurt: Campus.

Gruppe für Wissenschaft und Forschung: Homepage.

http://www.gwf-gsr.ch/deutsch/portrait/über_uns.htm, 29.9.2003.

Hagenbüchle, W. (2002). PISA - Der Bildungsmotor. NZZ Folio, 8.

Hard, F. X. (1974). Der eidgenössische Schulsekretär. Bestrebungen zu einer gesamtschweizerischen Schulkoordination im Jahre 1882. Zürich: Diss.

Heidenheimer, A. J. (1996). Bildungspolitik in der Bundesrepublik Deutschland, Japan und der Schweiz. «Innenpolitische» Staatsaufgaben im Wandel. In D. Grimm (Hrsg.), Staatsaufgaben (S. 585-611). Baden-Baden: Nomos Verlagsgesellschaft.

Klöti, E. (1903). Der Kampf um die eidgenössische Schulsubvention. Jahrbuch des Unterrichtswesens in der Schweiz, 15, 1-51.

Kussau, J. \& Oertel, L. (2001). Bildungsexpansion, Reform der Sekundarstufe I und Pädagogische Arbeitstellen. Schweizerische Zeitschrift für Bildungswissenschaften, 23, (1), 137-163.

Ladwig, J. G. (1994). For Whom this Reform? Outlining Educational Policy as a Social Field. British Journal of Sociology of Education, 15, (3), 341-363.

Meier, R .J. (1983). Der schweizerische Finanzausgleich im Hochschulwesen und im Bildungswesen allgemein. Zürich: Diss.

Meylan, J.-P. (1997). Die Maturitätsanerkennungsverordnung. In H. Badertscher (Hrsg.), Die Schweizerische Konferenz der kantonalen Erziehungsdirektoren 1897-1997 (S. 53-58). Bern: Haupt.

Müller-Solger, H. (1997). Bildung und Europa. Die EU-Fördermassnahmen. Bonn: Economica.

OECD (1991). Reviews of National Policies for Education - Switzerland. Paris: OECD.

OECD (2003). Beyond Rhetoric. Adult Learning Policies and Practices. Paris: OECD.

OECD (2003). Examen des tertiären Bildungssystems der Schweiz (deutsche Übersetzung des englischen Originaltextes). O.O.: OECD. http://www.gwf-gsr.ch/, 10.10.2003.

OECD: Homepage. http://www.oecd.org/about, 12.09.2003.

Osterwalder, F. (2000). Öffentliche Schule und Demokratie. Ein für den schweizerischen Liberalismus grundlegendes Verhältnis. In F. Oser \& R. Reichenbach (Hrsg.), Zwischen Pathos und Ernüchterung. Zur Lage der politischen Bildung in der Schweiz (S. 57-78). Freiburg: Universitätsverlag. 
PISA - ergänzende Perspektiven (2003). Themenheft. Schweizerische Zeitschrift für Bildungswissenschaften, 25, (1).

Scherrer, Ch. (2003). Neues von der GATS-Verhandlungsrunde. Forderungen zur Aufgabe staatlicher Verantwortung für die Hochschulen. Das Hochschulwesen, 51, (2), 60-67.

Schimank, U. (1988). Gesellschaftliche Teilsysteme als Akteurfiktionen. Kölner Zeitschrift für Soziologie und Sozialpsychologie, 619-639.

Schimank, U. (2002). Handeln und Strukturen. Weinheim \& München: Juventa.

Snell, L. (1840). Geist der neuen Volksschule in der Schweiz nebst den Hoffnungen, welche der Menschen- und Vaterlandsfreund daraus schöpft. St. Gallen: Wartmann.

Surdez, M. (1998). La mise à l'épreuve des gymnasiens. Les enjeux politiques et sociaux de la standardisation des examens et des certificats de maturité en Suisse entre 1870 et 1910. Bildungsforschung und Bildungspraxis, 20, (3), 432-445.

Troxler, P. I. V. (1830). Die Gesamthochschule der Schweiz und die Universität Basel. Trogen: s.n..

Troxler, P. I. V. (1848). Die Verfassung der Vereinigten Staaten Nordamerika's als Musterbild der Schweizerischen Bundesreform. Zum Neujahr 1848. Schaffhausen: Brodtmann.

Weber, K. (2000). Umrisse einer neuen Steuerungspraxis in der Bildungspolitik. Generalbericht und wissenschaftliche Analyse. In EDK (Hrsg.), Die Vielfalt orchestrieren (S. 195223). Innsbruck: Studien-Verlag.

Weisser, J. (1997). Traktanden, Geschäfte. Themen an den Plenarversammlungen der Schweizerischen Konferenz der kantonalen Erziehungsdirektoren 1897-1967. In H. Badertscher (Hrsg.), Die Schweizerische Konferenz der kantonalen Erziehungsdirektoren 1897-1997 (S. 59-102). Bern: Haupt.

Winter, D. (1998). Die eidgenössische Hochschule als nationales Projekt. Der Universitätsartikel und die Gründung der ETH. Bildungsforschung und Bildungspraxis, 20, (3), 375-388.

WTO: Homepage. http://www.wto.org/, 10.10.2003.

Schlagworte: Bildungspolitik, Bildungsföderalismus, internationale Organisationen, Globalisierung

\section{L'internationalisation de la politique fédérale en matière de formation}

\section{Résumé}

La question qui sera traitée dans cet article cherche à cerner comment les acteurs, figures importantes de la politique suisse en matière de formation et leurs programmes, se règlent sur l'internationalisation de la politique de formation. Il ressort que, aussi bien des acteurs centralisés, qu'au contraire, des acteurs orientés de la politique de formation prennent part à des projets internationaux. Le point de départ de la recherche est le développement de l'opposition historique sur le maintien de la différence politique entre un coordinateur vertical, puissant standardisateur, et un coordinateur horizontal. Alors que, historiquement, dans la plupart des champs politiques, la deuxième position était clairement dominante, la première s'est aussi développée de manière concurrentielle. Durant la phase d'expansion du système de formation, dans la deuxième moitié du 20ème 
siècle, le champ de la politique en matière de formation s'est progressivement différencié. De nouveaux acteurs ont fait leur apparition et les acteurs traditionnels se sont dotés d'équipes à visée scientifique. Par la suite, les deux orientations se sont atténuées de manière quasi programmable. L'apparition d'acteurs internationaux forts tels que l'OCDE et l'EU, la mise en place de Forums internationaux, les configurations et les stratégies mèneront, aussi bien pour les acteurs historiquement décentralisés que pour les acteurs orientés sur l'uniformisation, à des références communes.

Mots clefs: Politique de la formation, fédéralisme en matière de formation, organisations internationales, globalisation

\section{L'internazionalizzazione della politica formativa federalistica}

\section{Riassunto}

Ci si chiede come la politica formativa svizzera, con i suoi attori e i suoi programmi, reagisca all'internazionalizzazione della politica della formazione in generale. Una premessa è data dall'assunto che da parte svizzera ci sia una partecipazione a progetti internazionali. Inoltre, l'inchiesta parte dallo sviluppo del confronto storico tra una politica orientata verso una verticalizzazione e un'uniformazione e una politica a carattere orizzontale tesa ad assicurare le differenze. Mentre nella maggior parte dei contesti politici si può notare l'affermazione storica della seconda variante, nella politica del settore educativo si è sviluppata anche la prima variante. Il periodo di espansione del sistema educativo nella seconda parte del secolo scorso produce una progressiva differenziazione, con l'apparizione di nuovi attori e un'evoluzione di quelli tradizionali attraverso l'acquisizione di collaboratori scientifici. Essenziale sembra poi essere il fatto che l'affermarsi di attori a carattere internazionale come l'OCSE o l'UE con strategie proprie, diventa il punto di riferimento comune per entrambi gli orientamenti politici.

Parole chiave: Politica formativa, federalismo scolastico, organizzazioni internazionali, globalizzazione

\section{The Internationalisation of Federal Educational Policy}

\section{Summary}

The question of how agents, configurations of Swiss educational policy and their programmatic adjust to the internationalisation of educational policy is the central idea in this research paper. It is assumed that central as well as differentiated 
oriented agents in educational policy will participate in international projects. The starting point of the investigation is the development of historical contrasts between vertical, highly standardised and horizontal co-ordinators. Historically speaking the second orientation is clearly dominant in most policy fields, except that of educational policy where in addition to the second it also developed the first. The educational political field was increasingly differentiated during the expansion phase of the educational policy system in the second half of the 20th century. New agents show up and the traditional ones remain with their scientific oriented theories. At the same time both historical orientations exhausted themselves programmatically. The stronger appearance of international agents such as the OECD and the EU, thus the prominence of international forums, constellations and strategies becomes a commonly shared reference for the historically decentralised oriented agents aiming at difference as well as for the historically centralised agents aiming at unification.

Key words:educational policy, educational federalization, international organizations, globalization 